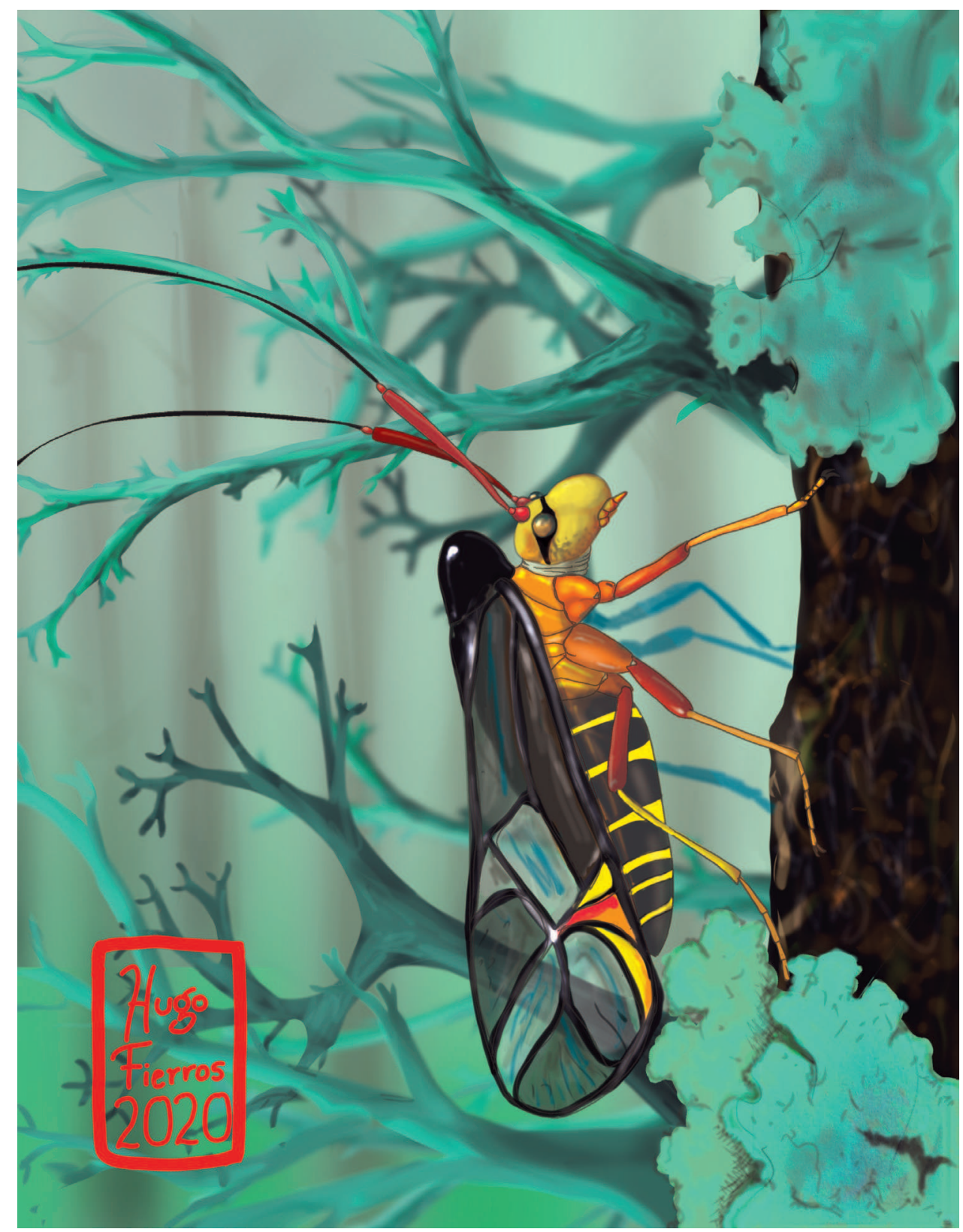

Dugesiana, Año 27, No. 2, julio 2020-diciembre 2020 segundo semestre de 2020), es una publicación semestral, editada por la Universidad de Guadalajara, a través del Centro de Estudios en Zoología, por el Centro Universitario de Ciencias Biológicas y Agropecuarias. Camino Ramón Padilla Sánchez \# 2100, Nextipac, Zapopan, Jalisco, Tel. 37771150 ext. 33218, http://148.202.248.171/dugesiana/index.php/DUG/index, glenusmx@gmail.com. Editor responsable: José Luis Navarrete-Heredia. Reserva de Derechos al Uso Exclusivo 04-2009-062310115100203, ISSN: 2007-9133, otorgados por el Instituto Nacional del Derecho de Autor. Responsable de la última actualización de este número: José Luis Navarrete-Heredia, Editor y Ana Laura González-Hernández, Asistente Editorial. Fecha de la última modificación 1 de julio 2020, con un tiraje de un ejemplar.

Las opiniones expresadas por los autores no necesariamente reflejan la postura del editor de la publicación.

Queda estrictamente prohibida la reproducción total o parcial de los contenidos e imágenes de la publicación sin previa autorización de la Universidad de Guadalajara. 
Artículo

\title{
Sinopsis de Hyporhicnoda Hebard, 1920 (Blattodea: Blaberinae: Hyporhicnodini), con nuevos datos de Hyporhicnoda ultima Grandcolas, 1993
}

\section{Synopsis of Hyporhicnoda Hebard, 1920 (Blattodea: Blaberinae: Hyporhicnodini), with new data of Hyporhicnoda ultima Grandcolas, 1993}

\section{Julio Cesar Estrada-Álvarez ${ }^{1,2}$, Carlo G. Sormani H. ${ }^{2,3}$}

${ }^{1}$ Museo Universitario de Historia Natural "Dr. Manuel M. Villada" Universidad Autónoma del Estado de México (UAEMex), Inst. Literario 100, Colonia Centro, Toluca, Estado México, México, C.P. 50000. ${ }^{2}$ Entomological Research, Metepec, Estado México, México. C. P. 52140. ${ }^{3}$ Instituto de Ecología, A.C., Ap. Postal 63, 91000, Xalapa, Veracruz, México. * micraten@yahoo.com.mx

\section{RESUMEN}

Es presentada una sinopsis del género Hyporhicnoda Hebard, 1920; proponiendose una nueva sinonimia dentro del género y una guía taxonómica para la separación de las hembras del género; son presentados nuevos datos y registros de H. ultima Grandcolas, 1993, describiendo el sexo femenino de la especie, hasta el momento desconocido.

Palabras clave: Distribución, Sinonimia, Neotrópico, Holotipo, Alotipo.

\begin{abstract}
A synopsis of the genus Hyporhicnoda Hebard, 1920 and a taxonomic guide for the separation of females from the genus, are presented. A new synonymy within the genus is proposed. New records of H. ultima Grandcolas, 1993 are presented, describing the female sex of the species, hitherto unknown.
\end{abstract}

Keywords: Distribution, Synonymy, Neotropic, Holotype, Allotype.

El género Hyporhicnoda Hebard, 1920 fue establecido con la especie tipo Rhicnoda reflexa Saussure y Zehntner, 1893, por monotipia (Hebard 1920: 98). El género en la actualidad está integrado por seis especies: $H$. carinata (Biolley, 1900), H. humilior Hebard, 1933, H. litomorpha Hebard, 1921, H. metae Hebard, 1921, H. reflexa (Saussure y Zehntner, 1893) e H. ultima Grandcolas, 1993, (Beccaloni 2014). De estas, para $H$. carinata y $H$. metae, se desconocen los machos, mientras que de H. ultima el sexo femenino es desconocido. Todos los registros del género son Neotropicales, siendo H. ultima la especie con el registro más septentrional del género, en Chiapas, México (Grandcolas 1993).

Dentro de la revisión de material de las colecciones CNIN e IEXA, encontramos varios ejemplares de Hyporhicnoda ultima Grandcolas, 1993, destacando ocho hembras. Al revisar la bibliografía nos percatamos que la hembras de H. ultima eran desconocidas y que los nuevos ejemplares representan nuevos registros que amplían su distribución. Aprovechando el tópico respecto al género Hyporhicnoda Hebard, 1920 y a la revisión de material tipo MHNG, ANSP, BMNH (fotos en Beccaloni, 2014) y MNHN (fotos); proponemos esta sinopsis del género Hyporhicnoda.

\section{MATERIAL Y MÉTODOS.}

Se revisaron ejemplares de las colecciones CNIN, INECOL y MHNG; así como fotos de MHNG, ANSP, BMNH y MNHN. Imágenes digitales fueron tomadas del material revisado, usando Photoshop CS4, para mejorar el contraste, brillo y tonos.

Las medidas se expresan en milímetros $(\mathrm{mm})$. El mapa se realizó usando la capa de mapa hipsográfico de INEGI (Mapa Digital de México 6.0), usando Photoshop CS4, para su edición. Abreviaturas. $\mathbf{D}=$ Descripción; $\delta=$ Macho; q $=$ Hembra; LT $=$ Localidad Tipo; reg. $\mathbf{n} .=$ Registro nuevo en bibliografía; sp. $\mathbf{n} .=$ Especie nueva.

\section{Colecciones.}

ANSP $=$ Academy of Natural Sciences of Philadelphia. Philadelphia, USA.

BMNH=British Museum of Natural History. London, UK. CNIN=Colección Nacional de Insectos, Instituto de Biología UNAM. CDMX, México.

IEXA=Colección Entomológica INECOL, Sede Xalapa. Xalapa, Veracruz, México.

MHNG=Muséum d'histoire naturelle de Genève. Genève, Suisse.

MNHN= Muséum national d'Histoire naturelle. Paris, France. 


\section{RESULTADOS Y DISCUSIÓN \\ BLATTODEA Brunner von Wattenwyl, 1882 \\ Superfamilia BLABEROIDEA Saussure, 1864 \\ Familia Blaberidae Brunner von Wattenwyl, 1865 \\ Subfamilia Blaberinae Brunner von Wattenwyl, 1865 \\ Tribu Hyporhicnodini Grandcolas, 1993}

Hyporhicnoda Hebard, 1920

Hyporhicnoda Hebard, 1920: 98.

Hyporhicnoda Roth, 1970: 316 [Blaberini]

Hyporhicnoda Grandcolas, 1993: 202. [Hyporhicnodini].

Especie tipo: Rhicnoda reflexa Saussure y Zehntner, 1893 [=Hyporhicnoda reflexa]; por monotipia Hebard 1920: 98 .

Diagnosis (enmendada): El género consiste en Blaberinos con dimorfismo sexual acentuado; machos completamente alados, hembras ápteras; espinación del fémur I tipo $\mathrm{B}_{1}$ (sensu Roth 2003), margen antero-ventral portando una a cuatro espinas basales y una hilera de espínulas muy reducidas, la espina apical corta y robusta; tarsomeros I-IV con pulvillus presente; uñas simétricas y simples; arolium ausente. Machos con tegminas y alas rebasando el abdomen; sin tergitos modificados, ni glándulas tergales (sensu Roth 1969) (obs. pers.); lamina supra-anal emarginada medialmente, cercos de ocho segmentos, paraproctos asimétricos, paraprocto derecho con esclerito bien esclerosado y en forma de gancho, paraprocto izquierdo membranoso (similar a Neorhicnoda Grandcolas, 1992); lamina sub-genital asimétrica con cercis cortos, simples y unisegmentados; genitalia masculina con prepucio portando una franja de espinas y ápice de L2vm con mayor pigmentación (Grandcolas 1993, Roth 1970) y ápice en forma de cuchara, L2d con la unión conspicua (Roth 1970). Hembras Ápteras, con textura rugosa y carina, media-longitudinal, presente, ya sea continua o parcial en pronoto, mesanoto y metanoto; lamina supra-anal emarginada medialmente, con los bordes laterales ya sean paralelos o convergentes, cercos unisegmentados; lamina sub-genital semicircular.

Género similar: Relacionado con Neorhicnoda Grandcolas, 1992, separados por la ausencia de sutura coxal sinuosa en cara anterior de la Coxa I (presente en Neorhicnoda); y genitalia masculina; adicionalmente, los Machos de Hyporhicnoda son fácilmente segregados por el desarrollo completo de las alas (brachipteras en Neorhicnoda) y la morfología del pronoto masculino (Grandcolas 1992, 1993). Hembras de Neorhicnoda, sin tubérculos en los márgenes de pronoto, mesonoto ni metanoto; lamina sub-genital sin emarginación conspicua (obs. pers.).

NOTA: Hebard en 1921, sugiere enmendar la diagnosis del género, en cuanto a la carina media-longitudinal, supuestamente ausente en $H$. litomorpha e H. metae (Hebard 1921: 142), pero en ambas especies se presenta estas carinas con desarrollo más discreto que sus congéneres (ver Fig. 11 y 12).

Especies que contiene: Hyporhicnoda reflexa (Saussure y Zehntner, 1893) (ฏ̊o)(Nicaragua-Panamá); Hypor- hicnoda humilior Hebard, 1933 ( đ̊ํ) (Panamá); Hyporhicnoda litomorpha Hebard, 1921 (ฏ̊ํ) (Panamá, Colombia); Hyporhicnoda metae Hebard, 1921 (ㅇ) (Colombia); Hyporhicnoda ultima Grandcolas, 1993 ( đ̊ํ) (México).

Distribución: Netamente Neotrópical, con registros discontinuos en el sureste de México, Nicaragua, Costa Rica, Panamá y centro-norte de Colombia (Fig. 16), con la posibilidad de que este género tenga una distribución continúa desde el sureste de México hasta el Noroeste de Colombia, abarcando toda Centroamérica (Fig. 16). Biogeograficamente se encuentra en las provincias Golfo de México, Yucatán, Oriente de América Central, Chocó-Magdalena y Maracaibo de la Subregión Caribeña (Fig. 16).

\section{Clave para la separación de las hembras de Hyporhic- noda Hebard, 1920. \\ (Machos consultar Roth 1970, Grandcolas, 1993, macho de metae desconocido)}

1a. Carinas medio-longitudinales en el pronoto, mesanoto y metanoto inconspicuas y discontinuas; granulación dorsal poco evidente y sin gránulos en los márgenes posteriores (Figs. 11,12).

1b. Carinas medio-longitudinales en el pronoto, mesanoto y metanotobiendefinidasyconspicuas; granulacióndorsalmarcada y gránulos en los márgenes posteriores (Figs. 13-15) .. 3 2a. Pronoto con impresión; meso y metanoto con dos áreas sin granulaciones; pronoto con carina medio-longitudinal solo en la parte posterior (Fig. 12) (Fig. 11) .. H. litomorpha 2b. Pronoto, meso y metanoto sin impresión; pronoto sin carina medio-longitudinal (Fig. 12 ....................... H. metae 3a. Abdomen sin ángulos caudo-laterales (no aserrado) (Fig. 13) H. ultima 3b. Abdomen con ángulos caudo-laterales (aserrado) (Fig. $14,15)$. ... 4 4a. Lamina supra-anal ligeramente emarginada en la parte media(Fig. 14) ................................................. H. humilior 4b. Lamina supra-anal profundamente emarginada (Fig. 15) H. reflexa

Hyporhicnoda ultima Grandcolas, 1993 (ðึำ) (Fig. 1-10, 13)

Hyporhicnoda ultima Grandcolas, 1993: 202; Fig. 17, 18 (D ${ }^{\text {A }}$ ) [sp. n.] [Mexique, Chiapas, Selva Lacandona, Chajul (LT)] Hyporhicnoda ultima Roth, 2003: 73

Hyporhicnoda ultima Estrada-Álvarez, 2013: 273

Hyporhicnoda ultima Beccaloni, 2014

Descripción de la hembra (Alotipo) (Fig. 2-9, 13). 29 largo total, 17 ancho máximo, completamente áptera; coloración ventral y dorsalmente de color marrón obscuro (chocolate); pronoto con el borde anterior más claro (Fig. 2, 3, 5 ), en menor medida que el macho (Fig. 1, 10). Pronoto (8 largo, 13 ancho), convexo, con los márgenes anteriores y laterales procurvos, rugoso, con quilla medial, margen posterior con tubérculos (Fig. 5, 6). Mesanoto (3 largo; 15 ancho) con una quilla en la parte medial, margen posterior con tubérculos (Fig. 6). Metanoto (3 largo; 16 ancho) con 
una quilla en la parte medial, margen posterior con tubérculos (Fig. 6). Cabeza completamente debajo del pronoto, de forma semi-redonda y rugosa; ojos compuestos reniformes con ambos lóbulos subiguales, distancia interocular 1; ocelos representados por dos manchas ocelares claras, distancia interocelar 1.1; antenas filiformes con el ápice aclarado; distancia entre escapos de 1.4, escapo subigual a tercer segmento antenal, segundo segmento reducido, los segmentos restantes subiguales a lo largo de las restantes (Fig. 7). Pata I, fémur con espinación tipo $\mathrm{B}_{1}$ : tres espinas cortas y robustas, seguidas de una hilera de espínulas muy cortas, terminando con una espina apical corta y robusta; tibia I claramente robusta, subtriangular, más ancha en el ápice, con nueve espinas fuertes y aserradas confinadas a la parte media apical. Pata II, fémur con tres espinas robustas y cortas sin espinación entre ellas, terminando con una espina apical corta y robusta; tibia II delgada cuatro veces más larga que ancha. Pata III, fémur con tres espinas robustas y cortas sin espinación entre ellas, terminando con una espina apical corta y robusta; tibia III delgada, ocho veces más larga que ancha, espinación a todo lo largo; tarsomeros de las patas 1-4 con pulvillus conspicuo; uñas simétricas y simples, sin arolium. Abdomen dorsalmente granular con los márgenes posteriores con tubérculos, decreciendo en número y tamaño en los tergitos posteriores; ventralmente los segmentos son menos granulares. Lamina supra-anal semicircular, medialmente emarginada, cercos unisegmentados (Fig. 8). Lamina sub-genital subtriangular (Fig. 9).

Diagnosis: Machos se segregan de sus congéneres por la morfología de la genitalia. La hembra de H. ultima es la única del género en contar con la combinación de caracteres: I) cuerpo dorsalmente con gránulos en los márgenes posteriores; Carinas medio-longitudinales, en el Pronoto, Mesanoto y Metanoto, incompletas; Bordes laterales de los tergitos no aserrados.

NINFAS: Machos y hembras similares a la hembra adulta.

Variación: Largo total ( $q$ q promedio $28, \mathrm{n}=8$. $\overbrace{}^{\lambda} \overbrace{}^{\lambda}$ promedio 25, $\mathrm{n}=10$ ).

Bionomía: Esta especie es gregaria en estadios juveniles de ambos sexos y hembras adultas, los ejemplares de Montes azules, fueron colectados y observados bajo la hojarasca al pie de un árbol; los machos adultos fueron hallados perchando sobre troncos de árboles vivos; similar a lo expuesto en Bell et al., 2007; Schal y Bell, 1986.

Material tipo: $1 \widehat{ }$ Holotipo y ${ }^{\wedge}$ Paratipo [CHIS, Selva Lacandona, Chajul; 1986-11-06/1986-11-13; Amédégnato C. y Poulain S. Cols.] (MNHN-EP-EP1234 Holotipo; MNHN-EP-EP1235 Paratipo). Disponible en: www.mnhn.fr (fotos revisadas).

Otro material revisado: $(18 ; 9 \hat{\jmath} \widehat{\partial}, 7$ 우, 2 juv.). 19 Alotipo MÉXICO, Chiapas, Mpio. Marqués de Comillas, Reserva de la Biosfera Montes Azules. Sel. Alta inundable; jul.-Oct. 2006; F. Escobar (91²9' 57.4” W, 16 49' 59.37'

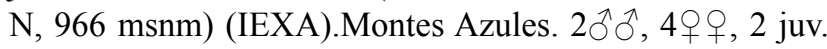
Chajúl: Estación Biológica de la Reserva de la Biosfera
Montes Azules; Junio-Octubre 2006; Sormani, H.C.G. Col. (IEXA). 2 우 Estación Biológica UNAM; Chajúl, Montes

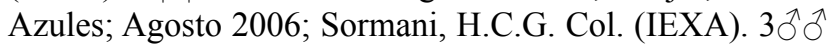
México, Ver, Est. Biol. de los Tuxtlas; 16/XI/1969; J. L.

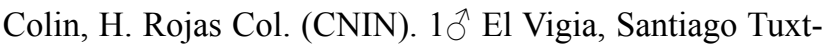
la, Ver.; 02/V/1968; Anónimo (CNIN). $1 \delta^{\widehat{T}}$ Coconá, Teapa, Tab.; 11/IV/1960; Anónimo (CNIN). $10^{\widehat{\lambda}}$ Central Chidera, El Lechugal, Camp.; 15/I/1953; Anónimo (CNIN). 1 + México, Chiapas, Marques de Comitán, Playón de la Gloria, Rio Lacantún, Alt. 151m. 16.1 ${ }^{\circ} 55^{\prime} 78^{\prime \prime} \mathrm{N}, 90.9^{\circ} 0^{\prime} 5^{\prime \prime} \mathrm{O}$; 12-15/XII/2012; V. Rivera et al. Cols. (CNIN).

Foto-registros: $\widehat{O}$ Belice, Distr. Stann Creek (17 01' 54.9", N88 31'09.7' W, 100 msnm); 13/V/2018; Foto de: Jordan Satler (Fig. 7) (Naturalista). $\bigcirc^{\wedge}$ Belice, Hickatee Cot-

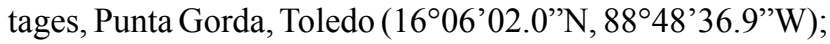

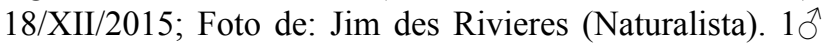

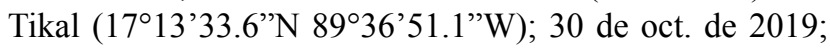
Foto de: Robert "redge91" (Naturalista).

Distribución conocida: México, Edo. Chiapas, Mpio. Marqués de Comillas, Loc. Selva Lacandona, Estación Científica Chajul [=Boca de Chajul] (Grandcolas 1993), [Localidad enmendada] $\left(90^{\circ} 55^{\prime} 30.17\right.$ ' W, $16^{\circ} 66^{\prime} 58.57^{\prime}$ " $\mathrm{N} ; 159 \mathrm{msnm}$ ), originalmente CHIS. Selva Lacandona. Chajul [etiqueta Holotipo].

NUEVOS REGISTROS: MÉXICO, Campeche, Mpio. Escárcega, El Lechugal. CHIAPAS, Mpio. Marqués de Comillas, Playón la Gloria en el Rio Lacantún y Reserva de la Biosfera Montes Azules. Tabasco, Mpio. Teapa, Coconá. Veracruz, Mpio. San Andrés Tuxtla, Estación de Biología Tropical Los Tuxtla; Mpio. Santiago Tuxtla, Cerro del Vigía de Abajo [Vigía Cooperativa]).

PRIMEROS REGISTROS NACIONALES: BELICE, Toledo (Hickatee Cottages, aprox. $1.5 \mathrm{~km}$. al Oeste de Punta Gorda); Stann Creek (Fig. 10). GUATEMALA, Depto Petén, Parque Nacional Tikal.

Biogeografía: Los registros están dentro de la provincia del Golfo de México y Yucatán (Fig. 17).

Nota: Los registros para Veracruz, Mpios. San Andrés Santiago Tuxtla son los registros más septentrionales de $H$. ultima y del género (Fig. 16, 17).

\section{Hyporhicnoda litomorpha Hebard, 1921 \\ (숭) (Fig. 11)}

Hyporhicnoda litomorpha Hebard, 1921: 139; Lam. X, Fig. 31-32 (DốP) [sp. n.] [Villavicencio, Intendencia del Meta, Colombia Elevation, 1400 feet (LT)]

Hyporhicnoda litomorpha Hebard, 1929: 372 [reg. n. Chiriqui Volcano, Panama]

Hyporhicnoda litomorpha Hebard, 1933a: 22 [reg. n. Antioquia, Colombia]

Hyporhicnoda litomorpha Hebard, 1933b: 117 [reg. n. Paraíso, Barro Colorado Island, San Blas, Panama]

Hyporhicnoda lithomorpha(sic) Apolinar, 1937: 134; Fig. 13ภ̄ [Villavicencio]

Hyporhicnoda litomorpha McKittrick, 1964: 17, 38, 39, 63 (욱)

Hyporhicnoda litomorpha Princis, 1967: 655

Hyporhicnoda litomorpha Roth, 1970: 316; Fig. 30

Hyporhicnoda litomorpha Salazar, 2001: 42 [reg. n. ME: CAL- 
DAS: Manizales]

Hyporhicnoda litomorpha Vélez, 2008: 26

Hyporhicnoda litomorpha Beccaloni, 2014

Distribución: Panamá, Prov. Chiriquí, Loc. Volcán Chiriquí [=Barú]; Prov. Panamá, Loc. Paraíso, Isla Barro Colorado; San Blas. Colombia, Villavicencio, Intendencia del Meta, Antioquia, Manizales (Apolinar 1937; Hebard 1921, 1929, 1933a, 1933b; Salazar, 2001).

Material revisado: $(2 q q) .1 q$ Holotipo Villavicencio, Colombia; 1919; A. María Col.; (ANSP-8722. Fotos de Heidi Hopkins, disponibles en http://cockroach.speciesfile. org). 19 Cocle, Cocle, PA; Fotos: John Garrett (Naturalista).

Hyporhicnoda metae Hebard, 1921

(ㅇ) (Fig. 12)

Hyporhicnoda metae Hebard, 1921: 138; Lam. V, Fig. 29, 30 $(\mathrm{D}$ 六 [sp. n.] [Villavicencio, Intendencia del Meta, Colombia Elevation, 1400 feet. LT]

Hyporhicnoda metae Apolinar, 1937: 134 [comparación con $H$. litomorpha]

Hyporhicnoda metae Princis, 1967: 655

Hyporhicnoda metae Vélez, 2008: 26

Hyporhicnoda metae Beccaloni, 2014

Distribución: Colombia, Depto. del Meta, Villavicencio.

Nota: Intendencia del Meta equivale en la actualidad al Departamento del Meta.

Material revisado: $(3 q q) .1 q$ Holotipo Villavicencio, Colombia; XII/1918; A. María Col. (ANSP- 8723. Fotos de Heidi Hopkins, disponibles en http://cockroach.speciesfile.org). 1 ㅇ Paratipo Villavicencio, Colombia; XII/1918; A. María Col (MNHN-EP-EP1237. Disponible en: www. mnhn.fr.). 19 Topotipo Villavicencio, Colombia; XII/1918; A. María Col. (MHNG).

\section{Hyporhicnoda humilior Hebard, 1933 (ํํㅇ)}

(Fig. 14)

Hyporhicnoda reflexa Hebard, 1920: 100; Lam. V, Fig. 9 [der. err.] [parte \& Porto Bello, Panamá, Sensu Hebard, 1933b: 119]

Hyporhicnoda humilior Hebard, 1933b: 118 (Dへ̂우) [sp. n.] [Gatún, Canal Zone, Panama (LT); Barro Colorado Island; Trinidad River; Porto Bello, Panama]

Hyporhicnoda humilior McKittrick, 1964: 17, 42, 43, 82; Fig. $180 \mathrm{~A}$

Hyporhicnoda humilior Princis, 1967: 655

Hyporhicnoda humilior Beccaloni, 2014

Distribución: Panamá, Prov. Colón, Loc. Gatún, Portobelo; Prov. Panamá, Loc. Isla Barro Colorado, Río Trinidad (Hebard 1920, 1933b).

Material revisado: $(1+$ ). 1 q Holotipo Gatún, Panamá; VII/28-VIII/05/1916; D. E. Harrower Col. (ANSP-8721. Fotos de Heidi Hopkins, disponibles en http://cockroach. speciesfile.org).
Hyporhicnoda reflexa (Saussure y Zehntner, 1893)

(ぷ+) (Fig. 15)

Rhicnoda reflexa Saussure y Zehntner, 1893: 68; Lam. IV, Fig. 35, (D $\left.{ }^{\Uparrow}\right)$ [sp. n.] [Nicaragua, Chontales (LT)]

Rhicnoda reflexa Saussure, 1895: 362

Rhicnoda reflexa Finot, 1897: 193

Rhicnoda carinata Biolley, 1900: 46 [sp. n.] [Tierras calientes; Turrialba, Las delicias (Santa Clara) La Virgen (Sarapiqui), Costa Rica; LT no definida] [Nombre provisional, ver notas] sinonimia nueva

Rhicnoda carinata Scudder, 1901: 282

Rhicnoda reflexa Scudder, 1901: 282

Rhicnoda reflexa Kirby, 1904: 124

Rhicnoda reflexa Shelford, 1910: 9

Hyporhicnoda reflexa Hebard, 1920: 8, 100; Lam. V, Fig. 8 [comb. n.] [parte Fig. 9 reg. Panamá =H. humilior, Sensu Hebard, 1933b: 118] [Ujurras de Terraba, head of Rio Ceibo, Costa Rica]

Hyporhicnoda reflexa Hebard, 1933b: 118 [reg. n. Changuinola District, Bocas del Toro, Panama]

Hyporhicnoda reflexa Princis, 1967: 655

Hyporhicnoda? carinata Princis, 1967: 655 [comb. n.]

Hyporhicnoda reflexa Roth, 1970: 3326; Fig. 112-114 [reg. n. Turrialba, Costa Rica]

Hyporhicnoda reflexa Fisk, 1971:440 [GCTE: Tilaran '64. PUNT: Ujarras de Terraba, head of Rio Ceibo. SJOS: $15 \mathrm{Km}$. No. of Quepos, San Jose]

Hyporhicnoda carinata Fisk, 1971: 440 [ALAJ: Las Delicias, Santa Clara. CART: Turrialba. HERD: La Virgen, Rio Sarapiqui] Hyporhicnoda reflexa Schal y Bell, 1986: 413, 414, 423

Hyporhicnoda reflexa Maes, 1992: 23

Hyporichnoda(sic) reflexa Bell et al., 2007: 41

Hyporhicnoda reflexa Beccaloni, 2014

Hyporhicnoda carinata Beccaloni, 2014

Al comparar ejemplares de $H$. carinata y $H$. reflexa, concluimos que ambas especies son sinonimias. En uso del principio de prioridad, se establece como sinonomia senior a Hyporhicnoda reflexa (Saussure y Zehntner, 1893) sobre Hyporhicnoda carinata (Biolley, 1900) sinonimia junior.

NOTAS: Rhicnoda carinata, es un nombre provisional propuesto por Biolley,en 1900, siendo la especie atribuible a Saussure, según el propio Biolley (1900: 46) “...por la especie inédita R. carinata, Sauss.,...”; Biolley, no provee descripción alguna. El material referido por Saussure y Zehntner (1893) y Biolley (1900), es el mismo.

Distribución: Nicaragua, Depto. Chontales (LT). Costa Rica, Prov. Alajuela. Loc. Las delicias (Santa Clara); Prov. Cartago, Loc. Ujarrás; Loc. Turrialba; Prov. Guanacaste, Loc. Tilaran; Prov. Puntarenas, Loc. Ujarras de Terraba, nacimiento Rio Ceibo; Prov. Heredia, Loc. La Virgen (Sarapiqui); Prov. San José, Loc. 15 Km. No. of Quepos, San José. Panamá, Prov. Bocas del Toro, Changuinola (Biolley 1900; Fisk 1971; Hebard 1920, 1933b, 1933b; Roth 1970; Saussure y Zehntner 1893).

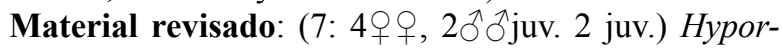

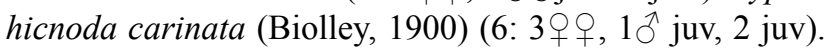
Costa Rica (MHNG). Rhicnoda reflexa Saussure y Zehntner, 1893 (2: 1ôjuv, 1q). 1ð̂ juv Chontales, Nicaragua; Janson Col.; (BMNH(E)\#878054, disponibles en Beccaloni, 2014). 1 ㅇ $1 \mathrm{~km}$ este de Tuis de Turrialba entrada a mano 
izquierda enfrente Jasec, Cartago Province, Tayutic, 30508, Costa Rica; Foto: driley (Naturalista).

Material adicional: Neorhicnoda maronensis (Hebard, 1921) (2: 1ठ, 19). OHolotipo. Guyane française, La Forestière; 1914; Lucien Chopard Col. (MNHN-EP-EP1240, Disponible en: www.mnhn.fr.). $ð$ Alotipo. Guyane française, Arataye; 1988-06-15; Desutter L. y Grandcolas P. Cols. (MNHN-EP-EP1238, Disponible en: www.mnhn.fr.).

\section{AGRADECIMIENTOS}

Externamos nuestra gratitud al Dr. Alejandro Zaldívar R., M. en C. Ma. Cristina Mayorga M. y M. en C. Enrique Mariño P. (CNIN-IBUNAM, UNAM, CDMX, México); Dra. Patricia Rojas, Biol. Antonio Ángeles y Dr. Delfino Hernández (INECOL, Veracruz, México); Dr. Peter Schwendinger y Dr. John Hollier (MHNG, Genève, Suisse), por las facilidades otorgadas para la revisión de material incluido en este estudio. A Jordan Satler, por permitirnos el uso de su foto (Fig. 10). A la Dra. Sonia Maria Lopes Fraga (Universidade Federal do Rio de Janeiro/ Museu Nacional-Entomologia, Rio de Janeiro, RJ, Brazil) y al Biol. Reinier Núñez Bazán, Centro de Investigación en Biodiversidad y Conservación, Universidad Autónoma del Estado de Morelos. Cuernavaca, Morelos, México; por sus comentarios y correcciones, que enriquecieron este trabajo. A Entomological Research por financiar este proyecto.

\section{LITERATURA CITADA}

Apolinar, M. 1937. Especies nuevas y observaciones diversas sobre Dermapteros y Ortopteros colombianos. Revista de la Academia Colombiana de Ciencias Exactas, Físicas y Naturales, 1(2): 132-137. doi.org/10.18257/ raccefyn. 665

Beccaloni, G.W. 2014 Cockroach Species File Online. 5.0/5.0. World Wide Web electronic publication. [Ultimo acceso 26 abril 2019], disponible en: <http://Cockroach.SpeciesFile.org>

Bell, W.J., L.M. Roth and C.A. Nalepa. 2007. Cockroaches: Ecology, Behavior, and Natural History. The Johns Hopkins University Press. Baltimore, USA.

Biolley, P. 1900. IV Ortopteres recogidos en Costa Rica desde 1890 a 1900. Informe Museo Nacional de Costa Rica 1899-1900: 40-49. doi.org/10.5962/bhl.title.10693

Estrada-Álvarez, J.C. 2013. Primera lista de las Cucarachas (Insecta: Dictyoptera: Blattaria) de México. Boletín de la Sociedad Entomológica Aragonesa, 53: 267-284.

Finot, A. 1897. Catalogue des Orthopteres de l'Amerique meridionale, derits jusqu'a ce jour 1895. Actes de La Societe Scientifique Du Chili, 7: 169-220.

Fisk, F.W. 1971. An annotated check list of Costa Rican cockroaches (Dictyoptera: Blattaria). Proceedings of the Entomological Society of Washington, 73(4): 431444.

Grandcolas, P. 1992. Paradicta n. gen. et Neorhicnoda n. gen., deux nouvaeux genres de Blaberinae (Dict., Blattaria, Blaberidae). Bulletin de la Société Entomologique de France, 97: 7-15.

Grandcolas, P. 1993. Monophylie et structure phylogénétique des (Blaberinae+Zetoborinae+ Gyninae+Diplopterinae) (Dictyoptera: Blaberidae). Bulletin de la Société Entomologique de France, 29(2): 195-222.

Hebard, M. 1920. The Blattidae of Panama. Memoirs of the American Entomological Society, 4: 1-148. doi. org/10.5962/bhl.part.13267

Hebard, M. 1921. Studies in Dermaptera and Orthoptera of Colombia Second paper. Transactions of American Entomological Society, 47:107-161.

Hebard, M. 1929. Previously Unreported Tropical American Blattidae (Orthoptera) in the British Museum. Transactions of the American Entomological Society, 55(4): 345-388.

Hebard, M. 1933a. Studies in the Dermaptera and Orthoptera of Colombia. Supplement to Papers One to Five. Transactions of the American Entomological Society, 59(1): 13-67.

Hebard, M. 1933b. Notes on Panamanian Dermaptera and Orthoptera. Transactions of the American Entomological Society, 59(2): 103-144.

Mapa Digital de México V6.3.0. INEGI. Disponible en: http://gaia.inegi.org.mx/mdm6

Maes, J.M. 1992. Catálogo de los Blattodea (Dictyoptera) de Nicaragua. I. Familias Polyphagidae y Blaberidae. Revista Nicaraguense de Entomología, 19: 21-28.

McKittrick, F.A. 1964. Evolutionary Studies of Cockroaches. Cornell University Agricultural Experiment Station, New York State College of Agriculture. New York, EE. UU.

Muséum national d'Histoire naturelle, Paris (France) (MNHN). Collection: Insects-Small orders y Odonates (EP) Disponible en: https://science.mnhn.fr

Princis, K. 1967. Pars 11. Blattariae: Suborbo [sic] Epilamproidea. Fam.: Nyctiboridae, Epilampridae. In: Beier, M. (Ed.). Orthopterorum Catalogus. Pars 11. W. Junk,'s-Gravenhage: 617-710.

Kirby, W.F. 1904. Fam. III Blattidae (PP 61-205) In: A synonymic catalogue of Orthoptera. Vol. I. Orthoptera Euplexoptera, Cursonia, et Gressoria (Forficulidae, Hemimeridae, Blattidae, Mantidae, Phasmidae). Trustees of the British Museum, Taylor and Francis (Eds.), UK, London.

Roth, L.M. 1970. The Male Genitalia of Blattaria. IV. Blaberidae: Blaberinae. Psyche, 77: 308-342. doi. org/10.5962/bhl.title.6745

Roth, L.M. 1969. The evolution of male tergal glands in the Blattaria. Annals of the Entomological Society of America, 62: 176-208.

Roth, L.M. 2003. Systematics and phylogeny of cockroaches (Dictyoptera:Blattaria). Oriental Insects, 37: 1-186. doi.org/10.1080/00305316.2003.10417344

Salazar, J. 2001. Blattodea de Colombia. Nuevas adicciones y rectificaciones a los mántidos de la primera parte (Insecta: Mantodea). Boletín científico Centro de Museos 
Museo de Historia Natural, 5:38-63. doi.org/10.17151/ bccm.2015.19.1.19

Saussure, H. y L. Zehntner 1893. Insecta-Orthoptera. Vol. I,

Fam. Blattidae. Biologia Centrali-Americana, (Porter) ed.: 1-112.

Saussure, H. 1895. Révision de la tribu des Panesthiens et de celle des Épilampriens (Orthoptères de la famille des Blattides). Revue Suisse de Zoologie, 3: 299-364. oi.org/10.5962/bhl.part.37722

Recibido: 19 septiembre 2019

Aceptado: 27 mayo 2020
Schal, C. and W.J. Bell. 1986. Vertical community structure and resource utilization in neotropical forest cockroaches. Ecological Entomology. 11:411-423.

Scudder, S.H. 1901. Alphabetical Index to North American Orthoptera Described in the Eighteenth and Nineteenth Centurie. Occasional papers of the Boston Society of Natural History, 6: 1-436.

Shelford, R. 1910. Blattidae (Orthoptera): subfamily Epilamprinae. Genera Insectorum. Fascicule 101:1-21.

Vélez, A. 2008. Checklist of Colombian cockroaches (Dictyoptera, Blattaria). Biota Colombiana, 9(1): 21-37.
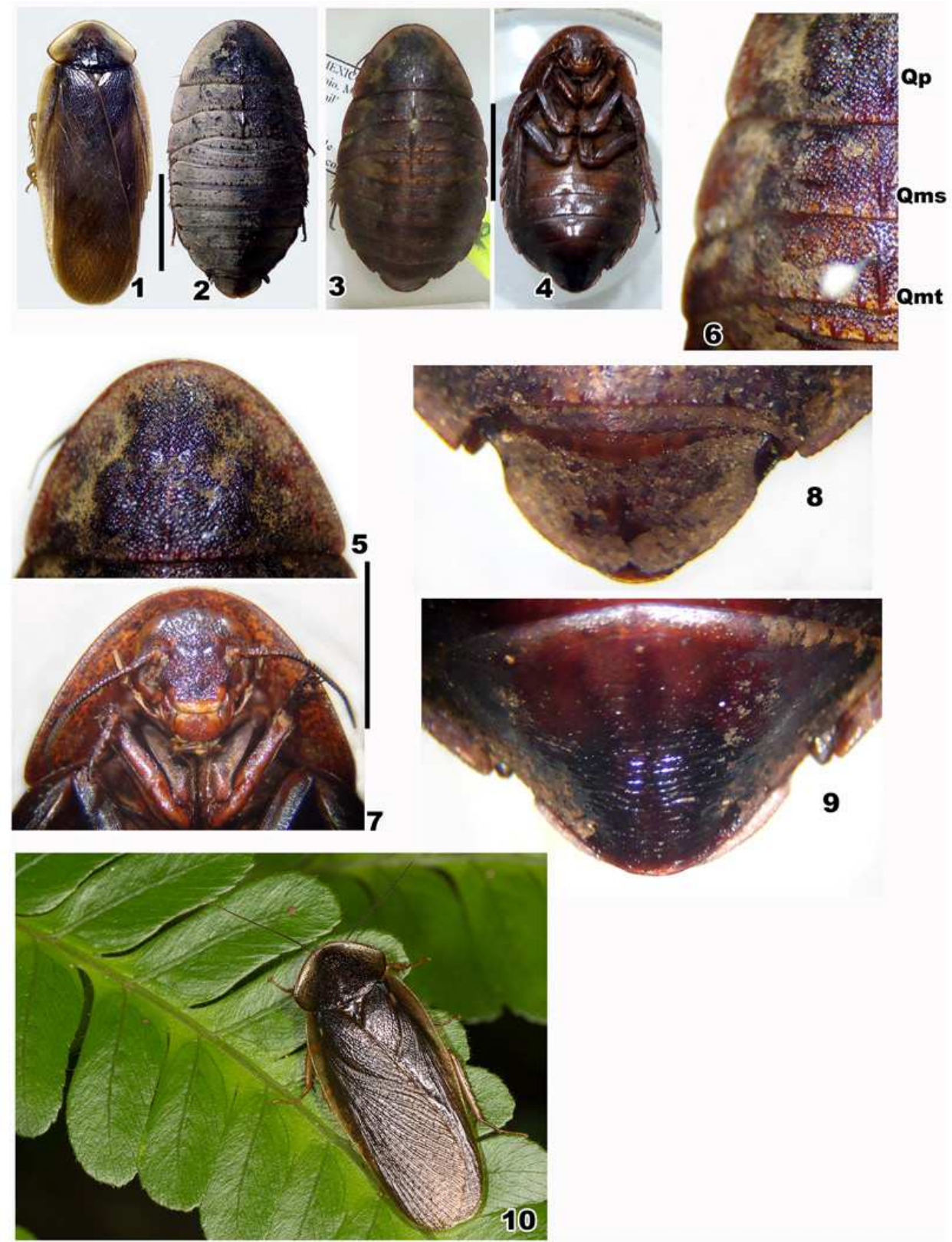

Figuras. 1-10. Hyporhicnoda ultima Grandcolas, 1993. 1, 10) Macho. 2-9) Hembra Alotipo Reserva de la Biosfera Montes Azules (IEXA). 1) Sintipo de Reserva de la Biosfera Montes Azules, CHIS, Vista dorsal (IEXA). 2) Hembra con la mitad izquierda con la cubierta terrosa, el lado derecho sin dicha cubierta. (2, 3) Vista dorsal, 4) Vista ventral, 5) Detalle del pronoto, 6) Detalle del pronoto-Metanoto, mostrando carinas, 7) Detalle de la cabeza, 8) Detalle de la Ls-a, 9) Detalle de Ls-g. 10) Macho in sutu Stann Creek District, Belize (Foto Jordan Satler). 


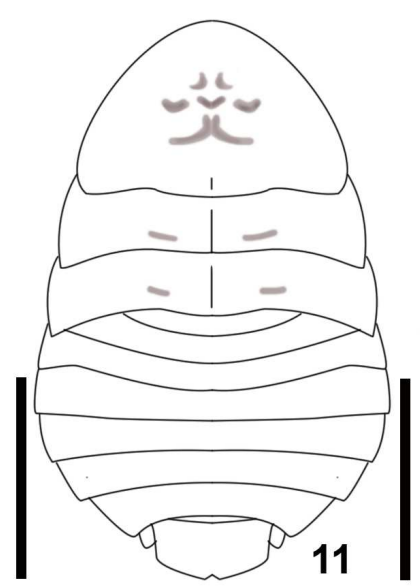

H. litomorpha

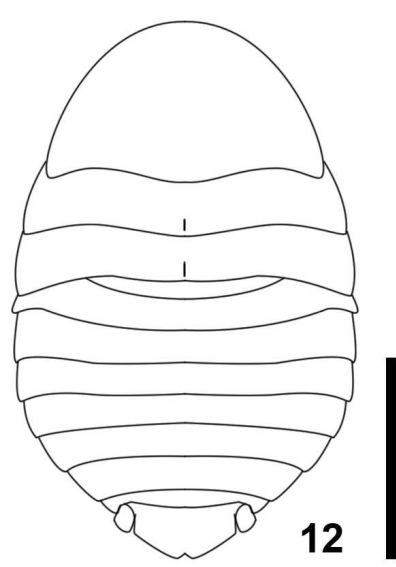

H. metae

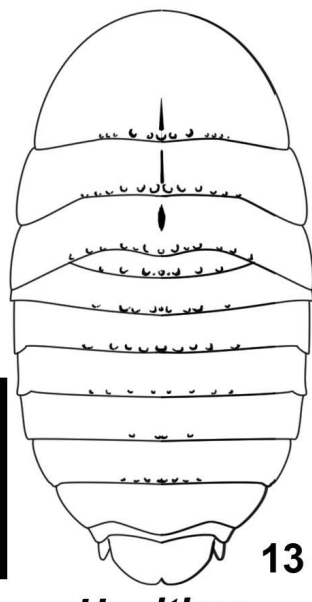

H. ultima

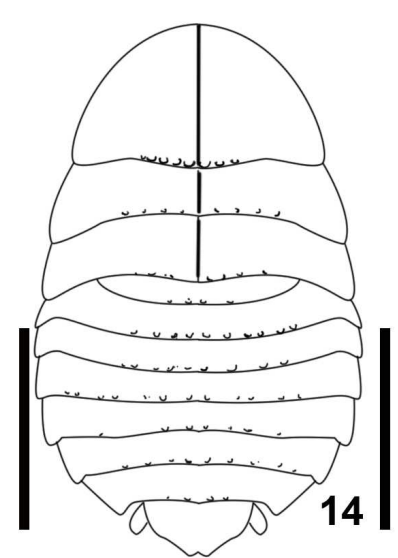

H. humilior

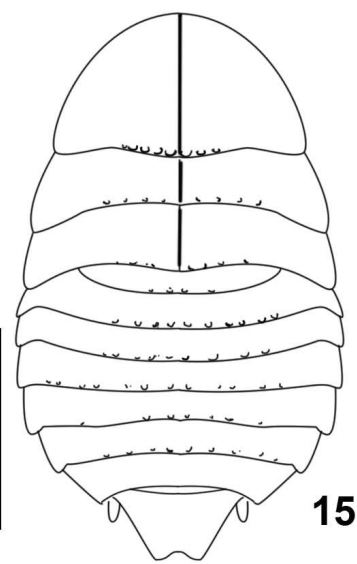

H. reflexa

Figuras.Fig. 11-15) Esquemas en vista dorsal de las hembras de Hyporhicnoda spp. 11) H. litomorpha Hebard, 1921; 12) H. metae Hebard, 1921; 13) H. ultima Grandcolas, 1993; 14) H. humilior Hebard, 1933; 15) H. reflexa (Saussure y Zehntner, 1893). 


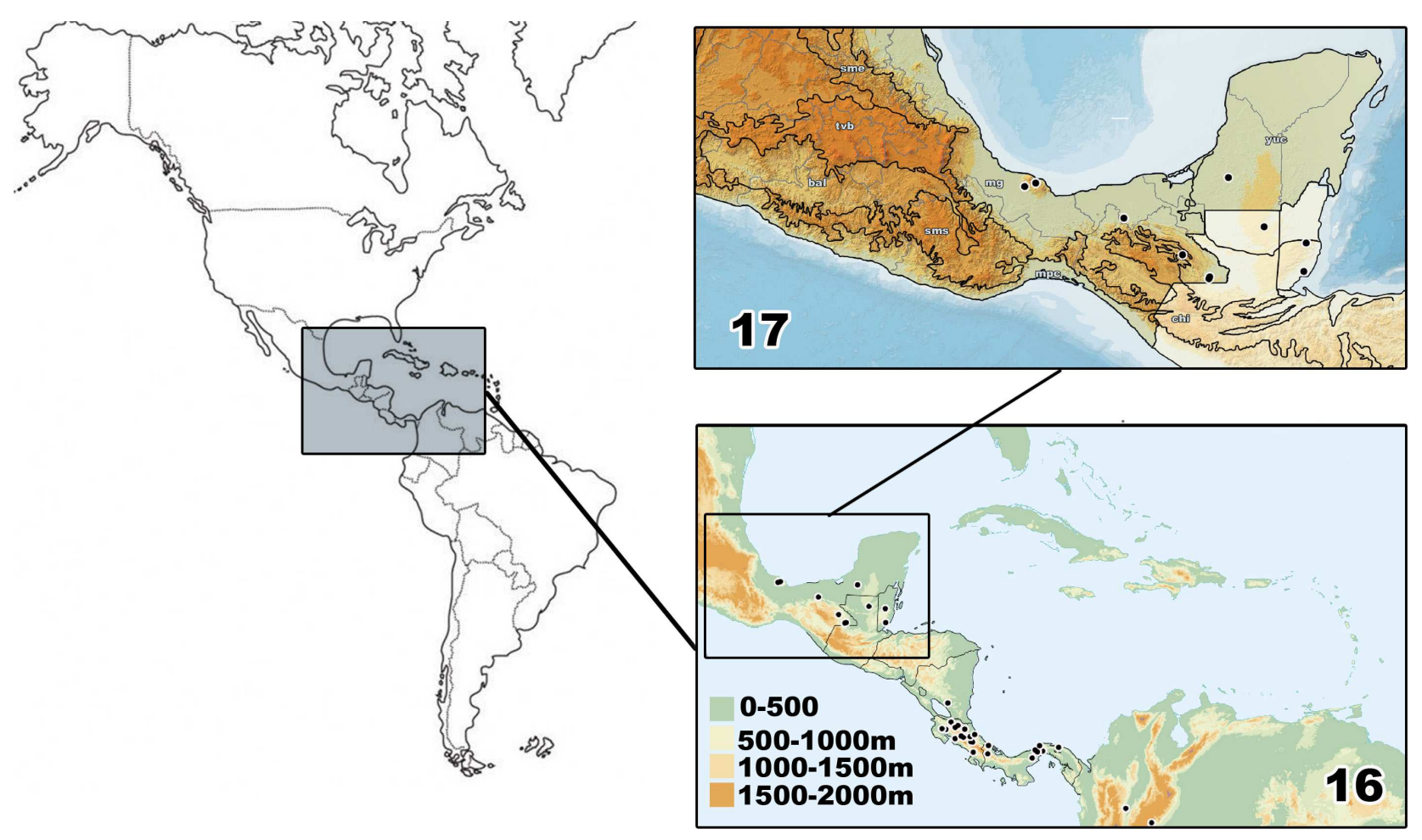

Figura 16) Distribución del género Hyporhicnoda Hebard, 1920. Figura 17) Distribución H. ultima en México y áreas limítrofes. Provincias biogeográficas. $\boldsymbol{b} \boldsymbol{a l}=$ Depresión del Balsas; $\boldsymbol{c h i}=$ Chiapas; $\boldsymbol{m g}=$ Golfo de México; $\boldsymbol{m} \boldsymbol{p} \boldsymbol{c}=$ Costas del Pacifico mexicano; $\boldsymbol{s} \boldsymbol{m} \boldsymbol{e}=$ Sierra madre oriental; $\boldsymbol{s} \boldsymbol{s} \boldsymbol{s}=$ Sierra Madre del Sur; $\boldsymbol{t} \boldsymbol{v} \boldsymbol{b}=$ Faja volcánica Transmexicana; $\boldsymbol{y} \boldsymbol{u} \boldsymbol{c}=$ Yucatán. 